\title{
Radioprotection : quel avenir ?
}

\author{
M. Bourguignon ${ }^{1,2, *}$, P. Bérard ${ }^{3}$, J.M. Bertho ${ }^{1, *}, \mathrm{~J}$ Farah $^{4}$, C. Mercat $^{5}$ et Comité éditorial de \\ Radioprotection \\ ${ }^{1}$ Institut de Radioprotection et de Sûreté Nucléaire (IRSN), Fontenay-aux-Roses, France. \\ 2 UVSQ - Université Paris-Saclay, Montigny-le-Bretonneux, France. \\ ${ }^{3}$ CEA, Fontenay-aux-Roses, France. \\ ${ }^{4}$ Hôpitaux Universitaires Paris-Sud, Le Kremlin-Bicêtre, France. \\ 5 Areva BS, Site de Tricastin, 26700 Pierrelatte, France.
}

Reçu le 8 février 2017 / Accepté le 9 février 2017

\begin{abstract}
Résumé - Durant les dernières années, Radioprotection, le journal de la Société française de radioprotection (SFRP), a évolué vers un journal international à comité de lecture. Cette évolution, simultanément à un changement récent dans le comité éditorial, a conduit à une réflexion sur les objectifs et les domaines d'intérêt du journal. Le point de départ de cette réflexion est une analyse par le comité éditorial des sujets d'intérêts actuels dans les différents champs de la radioprotection et les défis dans les domaines de recherche correspondants comme l'épidémiologie, la radiobiologie fondamentale, la dosimétrie biologique et physique, la radiotoxicologie, l'écotoxicologie et la radioprotection de l'environnement. Cet article présente les résultats de cette réflexion et ouvre la voie pour une évolution des objectifs et des domaines d'intérêt du journal Radioprotection.
\end{abstract}

Mots clés : radioprotection / expositions médicales / radon / radiobiologie / radiosensibilité / radiosusceptibilité / épidémiologie / dosimétrie

\section{Introduction}

Radioprotection, la revue internationale de la Société française de radioprotection (SFRP), s'est améliorée de façon continue ces dernières années. Elle est devenue une publication scientifique reconnue dédiée à la radioprotection, dotée d'un comité de lecture, référencée par un nombre croissant de bases de données bibliographiques et dont le facteur d'impact augmente progressivement. Le succès et la croissance ont apporté des bénéfices considérables mais ont parallèlement induit des défis majeurs. Un nouveau comité de rédaction a récemment été constitué et a entériné la mission que lui a confiée la SFRP de continuer à tendre vers l'excellence.

Dans la recherche des voies possibles de progrès vers un journal encore meilleur, il nous a paru utile d'identifier et de fixer par écrit les principaux thèmes de recherche et de discussion en radioprotection. Un sondage récent auprès du lectorat de Radioprotection nous a indiqué qu'il souhaite surtout des revues et des articles de synthèse sur les thèmes d'actualité. C'est le but de cet article d'identifier et de proposer des orientations pour les publications futures de Radioprotection.

\footnotetext{
* Auteurs de correspondance : michel.bourguignon@irsn.fr, jean-marc. bertho@irsn.fr
}

Nous pensons que les principaux sujets d'intérêt dans le domaine de la radioprotection et pour les lecteurs de Radioprotection sont ceux pour lesquels de nouvelles recherches et leurs résultats pourraient remettre en question l'approche et la gestion de la radioprotection. Dans le contexte actuel de limitation des ressources, les fonds alloués à la radioprotection sont restreints et proportionnés aux risques réels des radiations ionisantes par rapport aux risques induits par d'autres toxiques. Par conséquent, il est important de concentrer les efforts de recherche sur les questions clés de la radioprotection: "Une réponse approximative aux bonnes questions vaut beaucoup plus qu'une réponse précise à la mauvaise question » (John Tukey).

Cet article entend donc identifier les questions clés de la radioprotection qui peuvent être considérées comme prioritaires. L'intention est d'encourager l'échange de recherches et d'idées pertinentes au sein de la communauté de la radioprotection. Les manuscrits sur ces sujets sont donc les bienvenus dans Radioprotection, sans pour autant exclure des manuscrits dans d'autres domaines de la radioprotection.

\section{Justification scientifique}

Afin de discriminer ces questions clés, il nous a paru utile de revenir sur les origines de la radioprotection et sur son évolution. Nous pouvons identifier les étapes successives suivantes. 
Tout a commencé avec l'observation des effets cliniques des radiations ionisantes (RI), à savoir les brûlures cutanées et les cancers. Concernant les brûlures de la peau et d'autres effets déterministes majeurs, la responsabilité des RI est confirmée. Concernant les effets stochastiques (les cancers), l'établissement de la relation de cause à effet est plus complexe. Les études épidémiologiques ont beaucoup aidé à établir des corrélations fortes entre la fréquence d'apparition des cancers et les doses reçues pour des niveaux de dose au-delà de $100 \mathrm{mGy}$. Ces corrélations suggèrent fortement la responsabilité des RI dans l'apparition de cancers. Cependant, la démonstration que les RI sont la cause directe de l'apparition d'un cancer chez un individu donné n'a pas été faite jusqu'à présent, même pour les doses élevées de RI : il n'y a pas de marqueur biologique spécifique et fiable de cancer radio-induit ni aucun mécanisme spécifique et indéniable d'induction d'un cancer par les RI.

Les études épidémiologiques futures doivent être ciblées pour deux raisons:

- des cohortes épidémiologiques comportant des millions d'individus sont nécessaires pour augmenter la puissance statistique et réduire les incertitudes, en particulier dans la plage de doses inférieures à $100 \mathrm{mSv}$;

- le recueil des doses de RI n'est généralement pas d'une qualité suffisante puisque les données sont parfois recueillies rétrospectivement et sont souvent incomplètes.

Cependant, l'apport de l'épidémiologie a été primordial pour augmenter les connaissances sur les effets des RI sur la santé pour une large gamme de doses, au-dessus de $100 \mathrm{mGy}$. Elle a ouvert la voie à une gestion efficace de la radioprotection.

Les trois principes fondamentaux de la radioprotection de la CIPR, à savoir la justification, l'optimisation et la limitation de dose, ont été très efficaces pour une gestion optimisée des expositions. Les doses reçues chez l'homme ont diminué sensiblement pour atteindre le niveau des très faibles doses. L'exposition des travailleurs utilisant les RI a ainsi franchement baissé au cours des années et la dose efficace est actuellement de l'ordre de $1 \mathrm{mSv}$ par an ou moins dans la plupart des pays: $96 \%$ des travailleurs français reçoivent moins de $1 \mathrm{mSv}$ par an (IRSN, 2016) quoique quelques travailleurs médicaux peuvent dépasser la limite de dose de $20 \mathrm{mSv}$. La limite de dose pour le public qui est rarement dépassée est plus basse que la variabilité de l'irradiation naturelle. Les limites de dose du système actuel de la radioprotection sont très basses et il n'est pas prévu de les modifier dans le futur.

Alors, quel est l'avenir de la radioprotection?

Les sujets clés de la radioprotection que nous avons identifiés appartiennent aux catégories suivantes:

- les principales expositions aux RI, et surtout celles qui peuvent être diminuées ou optimisées ;

- les questions de dosimétrie physique et biologique;

- les futures études épidémiologiques;

- la radiobiologie, qui bénéficie actuellement de nouveaux outils remarquables aux niveaux moléculaire, cellulaire et tissulaire ;

- les questions liées à l'environnement et à l'écotoxicologie ;

- et in fine l'évolution nécessaire du système de la radioprotection.

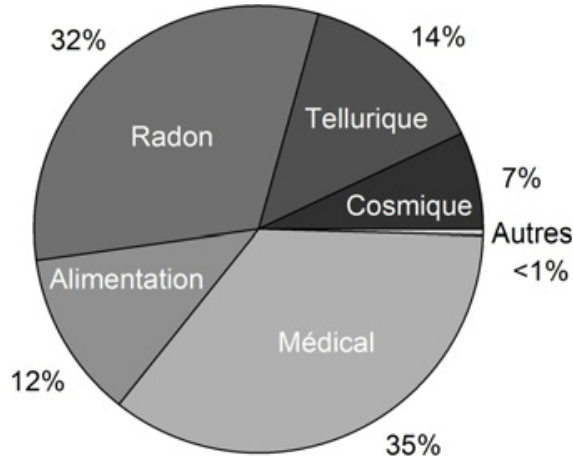

Fig. 1. Représentation schématique de l'exposition moyenne en France pour l'année 2015. Source : rapport IRSN 2015-0001 disponible sur www.irsn.fr.

\section{Les principales sources d'exposition humaine aux radiations ionisantes}

L'irradiation médicale et le radon sont les deux principales sources d'exposition de la population générale (Fig. 1). S'en préoccuper est une priorité non seulement parce qu'elles représentent les sources d'exposition les plus importantes et les plus fréquentes, mais également parce qu'il est possible de les contrôler dans une certaine mesure. C'est aussi le cas des expositions à la radioactivité naturelle dans le cadre d'activités industrielles. Les expositions et les expositions potentielles en cas d'accident doivent également être considérées.

Les expositions médicales diagnostiques des patients représentent en moyenne un tiers des expositions humaines annuelles. Elles augmentent partout dans le monde, à savoir d'un facteur 2 en France depuis les 10 dernières années (IRSN, 2014) et d'un facteur 6 aux États-Unis entre 1980 et 2006 (Herrman et al., 2012). Elles vont continuer à augmenter puisque l'imagerie médicale joue un rôle bénéfique majeur dans le diagnostic des maladies, l'orientation de la stratégie thérapeutique, le suivi des traitements et le traitement luimême à travers la radiologie interventionnelle et les actes radioguidés. D'autre part, le dépistage de certaines affections comme le cancer du sein par mammographie chez les femmes de 50 à 75 ans contribue de façon importante à la dose moyenne reçue par la population. En conséquence, l'exposition moyenne annuelle due aux expositions médicales continuera d'augmenter dans les prochaines années.

La variabilité des expositions médicales est importante entre les différents pays (Rapport européen Datamed II, 2014) mais le scanner X représente partout la majeure partie ( $>50 \%)$ des expositions des patients (UNSCEAR, 2008). Les doses efficaces médicales annuelles rapportées dans la littérature (par exemple $1,1 \mathrm{mSv}$ en Europe, $3 \mathrm{mSv}$ aux États-Unis) sont des valeurs moyennes obtenues sur l'ensemble de la population. Elles ne prennent donc pas en compte le fait que seulement une fraction de la population a une exposition médicale aux RI par an. La dose efficace annuelle d'origine médicale des patients exposés est donc plus élevée que la moyenne rapportée et est, de plus, très variable en fonction du nombre d'examens réalisés et de leur nature.

Les principes de justification et d'optimisation sont les principaux outils pour faire face à l'augmentation des 
expositions médicales. Par conséquent, leur utilisation doit être renforcée et leur application doit être étendue, par exemple, par la substitution des examens irradiants par l'IRM ou l'échographie, la révision des niveaux de référence diagnostique, les algorithmes de décision clinique, les innovations techniques des industriels pour diminuer les doses par exemple.

Une autre catégorie d'exposition médicale est la radiothérapie. Elle est utilisée avec succès (environ $80 \%$ ) partout dans le monde pour traiter environ $50 \%$ des cancers. Comme le risque de cancer croît avec l'âge et parce que la population mondiale vieillit, les expositions thérapeutiques aux RI vont augmenter. Les complications et les effets secondaires déterministes de la radiothérapie ne sont pas rares, affectant environ 10 à $15 \%$ des patients (Foray et al., 2016) et on observe environ $5 \%$ de cancers secondaires chez ces patients. Les nouveaux équipements et techniques (radiothérapie conformationnelle par exemple) permettent un meilleur ciblage de la dose à la tumeur, et en conséquence une escalade des doses et de nouveaux paradigmes de fractionnement. Ces progrès dus à l'innovation apportent des solutions intelligentes aux problèmes anciens mais soulèvent également de nombreuses questions nouvelles, par exemple l'augmentation importante de l'exposition des tissus normaux à de faibles doses de RI, la validité du modèle linéaire-quadratique pour la définition de nouvelles modalités de radiothérapie (fortes doses de protocoles d'hypo-fractionnement), l'augmentation du risque d'effets secondaires graves en cas d'erreurs balistiques.

L'exposition au radon représente également une part importante, d'un tiers en moyenne, de l'exposition de la population, quoique la majorité des individus ne soit exposée qu'à de faibles doses de radon. La dose absorbée et la dose équivalente aux poumons sont évaluées à 14,5 mGy/ $100 \mathrm{~Bq} \cdot \mathrm{m}^{-3}$ et à $290 \mathrm{mSv} / 100 \mathrm{~Bq} \cdot \mathrm{m}^{-3}$ respectivement. Les études épidémiologiques montrent que 10 à $15 \%$ des cancers des poumons sont associés à l'exposition au radon et que les effets de l'exposition au radon et du tabac (le facteur principal d'induction de cancers des poumons) sont plus qu'additifs. Au total, seulement 2 à $4 \%$ des cancers des poumons peuvent être attribués directement au radon seul.

Par ailleurs, l'exposition au radon est plus élevée dans les régions géologiques anciennes. La mauvaise ventilation des habitations favorise une pollution globale de l'air ambiant intérieur. En conséquence, la prévention de l'exposition au radon doit être considérée simultanément avec celle du tabac et des autres polluants atmosphériques intérieurs. Des recherches et des évaluations doivent être poursuivies dans ce domaine.

Les surexpositions accidentelles aux sources industrielles de haute activité, notamment celles utilisées pour le contrôle non destructif sont rares mais graves. Les conséquences sanitaires sont de deux types: syndromes d'irradiation aiguë et brûlures cutanées localisées.

Le traitement médical des surexpositions radiologiques a fait de remarquables progrès depuis 10 ans. Les syndromes d'irradiation aiguë bénéficient actuellement des cytokines et les greffes de moelle osseuse ne sont plus nécessaires et cette stratégie thérapeutique a fait l'objet d'un consensus international (Gorin et al., 2006). Les brûlures cutanées sont maintenant traitées par une combinaison de la chirurgie et de greffes de cellules souches (Lataillade et al., 2007). Les coopérations internationales doivent se poursuivre tant dans la compréhension des mécanismes mis en jeu que pour le suivi des patients. Deux points essentiels dans ce domaine. Il est important que le contrôle et la sûreté de ces sources industrielles soient améliorés à l'avenir. La recommandation de l'AIEA de mettre une marque de danger spécifique sur ces sources est un premier pas mais elle n'est pas sans risque et donc insuffisante. La réflexion sur la sûreté de ces sources industrielles doit être poursuivie. D'autre part, il importe de comprendre les mécanismes biologiques qui conduisent au succès des traitements par cellules souches mésenchymateuses. Ces traitements pourraient bénéficier d'une coopération internationale pour être davantage développés et étendus par exemple aux effets secondaires sévères de la radiothérapie.

Les expositions potentielles en cas de situation d'urgence ou d'accident concernent un grand nombre d'individus sur un large territoire et induisent des expositions aux faibles doses sur une longue période. Un intérêt particulier doit être porté aux travailleurs et aux sauveteurs qui peuvent être exposés à des doses relativement élevées de RI tant lors de l'accident que pendant les longues périodes de dépollution des sols et de traitement des déchets correspondants. Des avancées dans ce domaine aideront à retrouver la confiance du public.

Les sujets de recherche dans le domaine de la gestion de l'urgence notamment de son volet radioprotection sont:

- l'amélioration, l'harmonisation et la standardisation des modèles de dispersion et du comportement des radionucléides dans l'environnement;

- la poursuite des travaux concernant les radionucléides moins bien connus, leur comportement environnemental, leurs spécificités chimiques et physiques;

- l'amélioration et la standardisation des techniques et des procédures de mesure de la radioactivité et d'analyse des échantillons en laboratoire;

- l'amélioration des connaissances sur les effets sanitaires des expositions chroniques de longue durée aux faibles doses, internes et externes, en incluant les effets psychologiques ;

- l'identification des possibles interactions sanitaires entre les expositions aux RI et aux polluants chimiques;

- la préparation à la surveillance sanitaire nécessaire aux études épidémiologiques post-accidentelles : surveillance sanitaire préexistante, implication du public...;

- l'anticipation des questions liées à la vie en territoire contaminé: évacuation et retour, effets psychologiques, éthique, remédiation...;

- l'amélioration de la communication entre experts, le partage des connaissances avec les parties prenantes et la communication des incertitudes avec le public.

\section{La dosimétrie}

La dosimétrie est un outil essentiel de la radioprotection, les valeurs de doses étant indispensables à l'évaluation des expositions et essentielles aux études épidémiologiques. En conséquence, les doses doivent être déterminées avec précision et enregistrées rigoureusement.

Les doses reçues par exposition externe sont généralement bien évaluées. Cependant, dans de nombreuses situations, l'évaluation est plus complexe, par exemple pour les 
expositions prolongées, répétées, fractionnées, reçues en radiologie interventionnelle ou lors d'actes radioguidés. Au cours de la proton thérapie ou de la radiothérapie par des ions lourds et pour certaines installations nucléaires, une possible exposition externe aux neutrons de fuite dans certains postes de travail rend nécessaire l'utilisation de détecteurs appropriés et une vigilance particulière.

À l'inverse, les doses reçues par irradiation interne sont plus difficiles à évaluer correctement et avec précision. L'évaluation de la dose reçue par irradiation interne utilise les modèles biocinétiques de la CIPR pour les radionucléides inhalés ou ingérés, qui sont régulièrement affinés, puis des modèles dosimétriques de dose aux organes, aux tissus, aux cellules ou aux organites cellulaires. Mais l'établissement de certains de ces modèles nécessite des extrapolations à partir de radionucléides ayant des propriétés chimiques et physiques proches. De plus, comme les modèles biocinétiques dépendent de la spéciation du radionucléide, ils peuvent mal refléter le comportement de certains radionucléides "ciblés" utilisés en médecine nucléaire (radio-immunothérapie par émetteurs alpha par exemple) ou des radionucléides exotiques. Une attention particulière doit être apportée aux radionucléides impliqués dans les expositions professionnelles, accidentelles, naturelles et médicales impliquant le tritium organifié, le radon 222 , le plutonium 239, le strontium 90, l'iode 131 , le césium 137 , les isotopes de l'uranium et les radionucléides utilisés en radiothérapie par voie interne (radium 223, lutétium 177). L'évaluation de la fiabilité des modèles cinétiques et dosimétriques est donc une priorité.

Une autre avancée concerne la dosimétrie biologique, c'est-à-dire l'évaluation d'une dose à l'aide de paramètres biologiques. La dosimétrie biologique repose en partie sur l'évaluation des anomalies induites à l'ADN par les RI (aberrations chromosomiques, foci de $\gamma \mathrm{H} 2 \mathrm{AX}$, test des comètes, etc.). La sensibilité de ces tests a beaucoup augmenté ces dix dernières années, permettant l'évaluation d'une dose $\gamma$ interne dès 0,1-0,5 Gy. Cependant, l'abaissement des doses reçues par les travailleurs et la population et la demande croissante de fiabilité des résultats d'expositions (notamment dans le domaine médico-légal) imposent à la dosimétrie biologique toujours plus de sensibilité et de précision, ce qui représente un défi majeur.

\section{L'épidémiologie}

Comme cela a déjà été indiqué, l'épidémiologie est un pilier de la radioprotection. Les études épidémiologiques ont fourni des données très significatives qui ont été utilisées pour élaborer les recommandations actuelles en radioprotection.

Jusqu'aux années 1990, les survivants des bombardements nucléaires d'Hiroshima et Nagasaki et les patients traités par radiothérapie ont constitué la principale source d'information pour les études épidémiologiques. Elles ont montré une relation dose effet entre les effets sanitaires stochastiques et la dose pour des fortes doses délivrées à fort débit de dose. Elles ont aussi établi que le risque de cancer après exposition aux seuls RI à faible dose $(<100 \mathrm{mGy})$ et faible débit de doses $(<5 \mathrm{mGy} / \mathrm{h})$ est très petit puisqu'elles n'ont pas été capables de démontrer un risque.
Dans la dernière décennie, plusieurs études épidémiologiques ont fourni des résultats nouveaux pour les faibles doses et les faibles débits de dose: travailleurs de Mayak, résidents de la rivière Techa, travailleurs de l'industrie nucléaire, patients exposés en imagerie médicale, liquidateurs de Tchernobyl... Elles n'ont pas été capables de démontrer un risque significatif aux faibles doses jusqu'à présent mais les extrapolations semblent compatibles avec une relation linéaire sans seuil. Des études extensives donneront des résultats additionnels de grand intérêt.

\section{Que peut-on espérer de l'épidémiologie? Et par quels moyens?}

En application des théories statistiques, les études épidémiologiques augmentent leur puissance avec la taille des cohortes. L'étude australienne concernant les expositions pédiatriques au scanner $\mathrm{X}$ et portant sur des millions d'enfants (Mathews et al., 2013) et les études INWORKS combinant 3 cohortes et plus de 300000 travailleurs (Leuraud et al., 2015; Laurier et al., 2016) sont de bons exemples de confirmation. Puisqu'elles sont utiles, de telles études doivent être poursuivies, comme par exemple l'étude européenne EPI-CT du scanner X chez l'enfant qui combine 8 cohortes et est toujours en cours (Bosch de Basea et al., 2015).

Néanmoins, les études épidémiologiques sont biaisées du fait de données incomplètes concernant les doses, par exemple la non-prise en compte des doses médicales. Quelles sont les doses médicales reçues par les survivants des bombardements atomiques au Japon pendant les dizaines d'années de leur suivi médical ou par les travailleurs des études INWORKS pendant le suivi médical au cours de leur longue carrière professionnelle? Il y a beaucoup de raisons de penser que ces doses médicales peuvent être tout à fait significatives au point de pouvoir modifier les conclusions des études épidémiologiques. De combien, cela reste à déterminer. Pour la validité des études épidémiologiques futures concernant les populations concernées à Fukushima, leurs doses d'origine médicale devraient être enregistrées.

Les études épidémiologiques peuvent aussi être biaisées quand elles ne prennent pas en compte les expositions potentiellement significatives aux nombreux autres composants génotoxiques de l'environnement professionnel ou privé comme les pesticides, l'alcool, le tabac et autres polluants chimiques de l'atmosphère... Cela peut être le cas dans les situations post-accidentelles dans laquelle la population est exposée aux RI et à des polluants chimiques. Le risque des expositions combinées doit donc être évalué à l'avenir. Cela est essentiel pour l'évaluation des effets des faibles doses de RI puisque, plus la dose est faible, plus sa responsabilité potentielle dans la survenue d'un cancer, maladie complexe de survenue tardive due à une combinaison délétère de lésions de l'ADN, est faible (Weinberg, 2013).

En conséquence, il convient de clarifier les conclusions des études épidémiologiques au sujet de l'association des RI à faible dose avec un risque de cancer, et des RI comme risque de cancer parmi d'autres risques et la cause de la maladie. Des clarifications sont nécessaires et les interprétations erronées doivent être évitées!

À côté de l'augmentation de puissance des études épidémiologiques résultant de l'augmentation de la taille des cohortes, l'épidémiologie gagnera en force en se focalisant sur 
des cohortes sélectionnées sur la base de marqueurs biologiques, par exemple des marqueurs de la réponse individuelle potentiellement anormale aux RI. À cet égard, il conviendra d'associer aussi les investigations «omiques» (génomiques, protéomiques et autres) aux études épidémiologiques afin d'augmenter leur pouvoir analytique. De plus, ces nouvelles cohortes seront utiles pour clarifier la relation entre l'effet sanitaire et la dose: jusqu'ici, la relation linéaire entre l'effet sanitaire (cancer) et la dose est prouvée pour des doses supérieures à $100 \mathrm{mGy}$ mais la radiobiologie démontre une relation linéaire entre les lésions de l'ADN et la dose à partir de doses supérieures à 1 mGy (Rothkamm and Lobrich, 2003). Bien sûr, une lésion de l'ADN n'est pas suffisante pour créer un cancer mais il n'y a pas de cancer sans lésions de l'ADN et l'accumulation d'un certain nombre de lésions $(>10)$ est nécessaire. Pouvons-nous donc combler ce vide de connaissance entre les lésions de l'ADN et le cancer? Pouvons-nous étendre la relation linéaire entre les effets sanitaires et la dose à de plus bas niveaux de dose, au moins pour des sous-groupes de population?

Finalement, des résultats de l'épidémiologie suggèrent une association entre les expositions aux RI et des effets non cancéreux comme les maladies cardiovasculaires (Little et al., 2012). Le contrôle des facteurs de risque autres que les RI est nécessaire. De futures études épidémiologiques devraient fournir une confirmation dans les dix prochaines années.

\section{La radiobiologie}

La radiobiologie a historiquement ouvert la voie de la radioprotection. De nouveaux outils d'analyse aux niveaux moléculaire, cellulaire et tissulaire ont été développés. Ils ont induit des données suffisamment nouvelles pour modifier en profondeur notre compréhension des effets des RI et leur garder une place centrale dans la radioprotection. Ainsi, des recherches approfondies doivent être menées pour répondre aux nouvelles questions qui se posent et répondre à de nombreuses inconnues liées à la grande variété des expositions.

De grand progrès ont été réalisés dans le domaine de la radiobiologie au niveau fondamental notamment quand les RI sont utilisés comme agents perturbateurs. Les nouveaux outils d'exploration ont déjà apporté des résultats significatifs et en apporteront sûrement d'autres :

- Les nouvelles techniques mises au point ces dix dernières années ont permis un gain de sensibilité de détection des lésions de l'ADN d'un facteur 100 grâce à l'immunofluorescence des histones $\gamma \mathrm{H} 2 \mathrm{AX}$ lors des cassures doublebrin, visibles dès $1 \mathrm{mGy}$ d'exposition (Rothkamm and Lobrich, 2003). D'autres traceurs immunofluorescents ont été développés, permettant une analyse dynamique: la bonne protéine est-elle au bon endroit au bon moment (ATM, Rad 51, MRE11...) (Granzotto et al., 2016) ? Au total nous avons actuellement une évaluation fonctionnelle dynamique de la signalisation des dommages induits à l'ADN par les RI et des voies de leur réparation (DDR). Les résultats des recherches «omic» commencent à apporter de nouvelles connaissances sur les effets des RI et pourraient en particulier indiquer une signature radiologique des cancers après les très fortes doses de la radiothérapie (Behjati et al., 2016).
- L'hyperradiosensibilité aux faibles doses de RI (phénomène HRS), caractérisée par l'augmentation significative des morts cellulaires aux faibles doses d'exposition (10 $800 \mathrm{mGy}$ avec un pic vers $300 \mathrm{mGy}$ ) par rapport aux fortes doses (1 Gy) (Joiner et al., 2001), s'explique désormais par des perturbations de DDR. L'importance de HRS pour les doses couramment rencontrées reste à déterminer.

- En ce qui concerne l'effet non ciblé, les composés solubles qui véhiculent dans le voisinage les effets génotoxiques et clastogéniques restent à découvrir. De même, la fréquence et l'importance de l'effet bystander dans la réponse aux RI à l'échelle de l'organisme entier restent à définir.

- Une réponse anormale aux RI pourrait être beaucoup plus fréquente que nous ne le pensions (environ $20 \%$ de la population) (Foray et al., 2016). Cela soulève des interrogations en santé publique qui ne peuvent plus être négligées. La réponse individuelle aux RI semble présenter 3 aspects : 1- la radiosensibilité aux RI, qui induit des effets déterministes liés à l'augmentation des morts cellulaires et à la perturbation de l'homéostasie tissulaire après exposition aux fortes doses de RI (effets secondaires voire complications graves de la radiothérapie en l'absence d'erreur d'exposition); 2- la radiosusceptibilité aux RI caractérisée par la survie de cellules lésées (qui induit une tendance élevée à développer des cancers après exposition aux RI) et correspond peut-être à une tendance globale à développer des cancers. Ce dernier aspect souligne le rôle potentiel de la combinaison d'agents toxiques différents comme agents inducteurs d'anomalies clastogéniques et génotoxiques ; 3 - la radiodégénérescence individuelle, qui se traduit par des effets secondaires tardifs non cancéreux tels que les cataractes et les effets cardiovasculaires, semble également liée à des anomalies de DDR.

- Les mécanismes précis de la réponse individuelle aux RI commencent donc à être clarifiés avec l'implication de DDR mais beaucoup reste encore à découvrir.

Tous ces résultats confirment que les premières lésions induites par les RI se produisent au niveau de l'ADN cellulaire. Le nombre de lésions observées croît linéairement avec la dose reçue : 40 cassures double-brin de l'ADN et des centaines d'autres lésions de l'ADN sont créées pour une dose absorbée de $1 \mathrm{~Gy}$ et peuvent conduire à des lésions complexes. La qualité de la signalisation et la réparation correcte ou non de ces lésions sont le point central du devenir de la cellule lésée, y compris à faibles doses (Colin et al., 2011). Elles représentent le cœur de la future radiobiologie. Elles reflètent la variabilité des réponses individuelles aux RI (Joubert et al., 2008).

Toutes ces techniques nouvelles doivent être mises en jeu pour élucider les mécanismes fondamentaux et pour répondre à quelques interrogations récurrentes :

- existe-t-il une réponse différente suivant la dose et le débit de dose et/ou selon que l'irradiation est interne ou externe?

- quels sont les facteurs induisant une absence ou un retard de réponse au niveau des faibles doses et quelles en sont les conséquences?

- quelle est l'influence de l'âge au moment de l'exposition et existe-t-il une réponse différentiée en fonction du sexe?

- quelle est l'importance des effets non ciblés de l'exposition aux RI? 


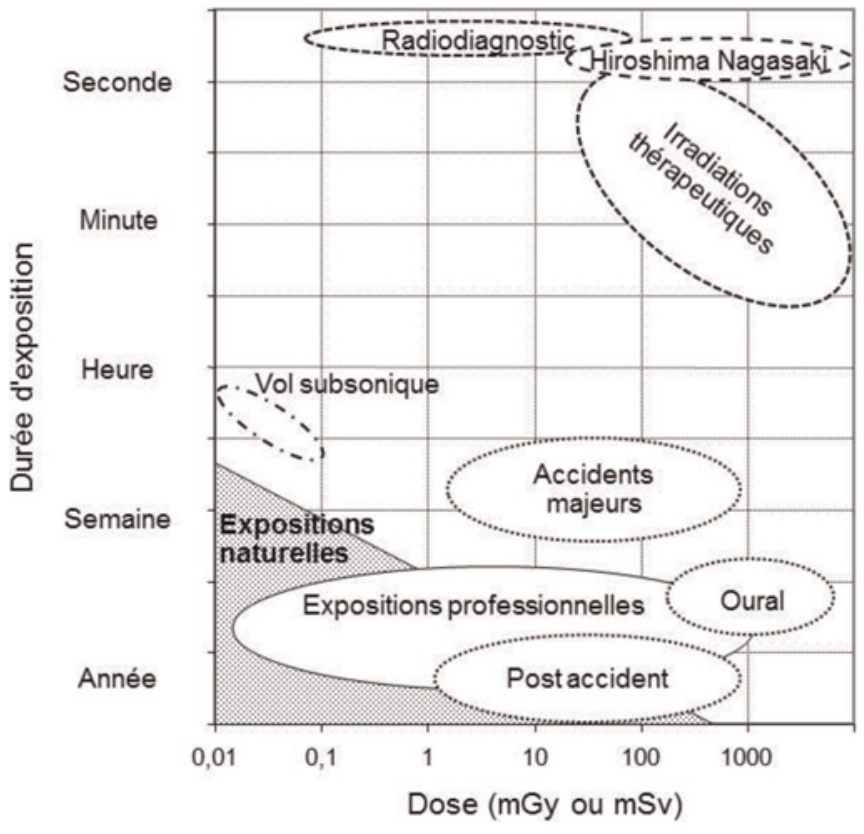

Fig. 2. Représentation schématique des différents types d'exposition en fonction de la dose et de la durée d'exposition.

- quel rôle joue l'environnement cellulaire et les réactions tissulaires en particulier métaboliques et inflammatoires?

Les expositions médicales, par le radon ou accidentelles correspondent à des niveaux de dose et à des débits de dose très différents (Fig. 2) (Hubert, 2003). Ils peuvent aussi être très différents des irradiations aiguës (par exemple celles des survivants des bombardements atomiques) qui servent de socle à notre connaissance sur les effets de RI. Il y a beaucoup d'autres types d'expositions qui sont importants en pratique clinique et pour lesquels il n'y a pas ou peu de résultats de radiobiologie: la répétition des doses dans des intervalles courts (par exemple le second cliché de la mammographie de dépistage), l'utilisation des agents de contraste qui induisent une augmentation importante de la dose absorbée, la répétition des investigations radiologiques dont les doses cumulées peuvent dépasser $100 \mathrm{mGy}$ correspondant au seuil d'apparition d'effets stochastiques, les réponses différentes en fonction de l'énergie $(30 \mathrm{kV}$ en mammographie versus $120 \mathrm{kV}$ pour le scanner $\mathrm{X}$ ) et les différentes réponses cellulaires liées aux nouveaux paradigmes de la radiothérapie. Les effets des expositions chroniques et continues ne sont pas si bien connus. Des recherches en radiobiologie doivent donc être menées pour explorer ces questions qui n'ont pas de réponse actuellement.

Les mécanismes biologiques et moléculaires fondamentaux de la réponse aux RI sont étudiés sur des animaux ou sur des cellules animales en culture, mais des travaux sur du matériel humain semblent indispensables parce que les cellules humaines ont un plus grand niveau de complexité en comparaison des cellules de rongeurs et que certains mécanismes moléculaires sont spécifiques aux cellules humaines. Des travaux sur des collections d'échantillons ou de matériel biologique humain (comme le registre américain USTUR [United States Transuranium \& Uranium Registries]) ou la collection COPERNIC de fibroblastes de patients radiosensibles (Granzotto et al., 2016) doivent être encouragés. De même des investigations par des méthodes multiples sur les mêmes échantillons permettront une compréhension plus fine et plus sûre des mécanismes mis en jeu.

Enfin il est utile de poursuivre les travaux de radiobiologie sur la faune et la flore qui participent à la radioprotection de l'environnement afin de comprendre les mécanismes et les spécificités de leurs réponses aux RI.

\section{Radioprotection et environnement}

La radioprotection de l'environnement s'est considérablement développée depuis les années 1960, rendant aujourd'hui disponibles des connaissances, des méthodes et des outils éprouvés en matière d'évaluation des risques radiologiques pour l'homme dans son environnement, et plus récemment pour l'environnement lui-même. De nombreuses questions concernant les expositions externes et internes, les modèles biocinétiques et dosimétriques ont déjà été évoquées dans cet article.

Initié depuis une dizaine d'année, le rapprochement entre les méthodes d'évaluation de l'impact dosimétrique et les méthodes d'évaluation des risques sanitaires (associés aux produits chimiques) constitue une avancée intéressante en matière d'harmonisation et d'optimisation des pratiques car les installations nucléaires sont aussi des usines chimiques et pour nombre d'entre elles, les évaluations des effets sanitaires pour les groupes de référence du public s'avèrent finalement plus élevées que l'impact dosimétrique. L'évaluation du risque combiné doit être poursuivie et doit évoluer en développant des outils simplifiés qui intègrent toutes les dimensions du risque (protection du public et protection des écosystèmes vis-à-vis des RI et des substances chimiques) au niveau approprié de complexité en application du principe de proportionnalité (adéquation des moyens au but recherché). Par ailleurs, il est indispensable de confronter l'évaluation du risque avec les observations de terrain sur la faune, la flore et la biodiversité pour contrôler la validité des modèles.

D'un point de vue opérationnel, des enjeux industriels majeurs en matière de radioprotection de l'environnement sont à venir du fait de chantiers de démantèlement en augmentation et de la mutation industrielle nécessaire d'un certain nombre de sites nucléaires. Ces nombreux chantiers doivent aujourd'hui déjà faire face à des difficultés grandissantes quant à la gestion des terres excavées du fait d'un foncier contraint. Le retour à l'état naturel, c'est-à-dire l'assainissement de la totalité des becquerels ajoutés, est le dogme incontournable mais sur le terrain, cet objectif vertueux est rarement atteignable: continuité des activités économiques déjà en place lors de l'arrêt d'une installation, contraintes techniques insolubles pour excaver les volumes conséquents mis en jeu, absence de capacités suffisantes des filières de stockage actuelles pour accueillir des terres excavées considérées comme des déchets nucléaires. La clarification des concepts vis-à-vis des déchets et leur gestion, y compris les seuils de libération, est nécessaire. Il faut collectivement progresser dans la recherche de critères d'assainissement et dans l'identification de solutions pragmatiques pour les opérateurs et acceptables par l'ensemble des parties. 
En conséquence, l'évaluation des risques, l'optimisation des rejets autorisés, les objectifs d'assainissement lors du démantèlement, la gestion post-accidentelle de territoires contaminés... sont différentes thématiques de radioprotection de l'environnement ouvertes à la discussion au sein de la communauté de la radioprotection et avec la société civile qui est très concernée par les questions d'empreinte environnementale. La démocratie participative rend indispensable le partage des points de vue, des pratiques et des retours d'expérience au niveau international et le partage des priorités sur les questions de radioprotection environnementale.

\section{Le système de radioprotection}

Le système de radioprotection développé et mis à jour par la CIPR (ICRP, 2007) depuis plus de 80 ans s'est montré robuste et opérationnel.

La présente revue a pour intention d'ouvrir la discussion dans les différents champs de la radioprotection. Ces discussions ont pour vocation d'ouvrir de nouvelles voies de réflexion pour le futur du système de radioprotection. Nous anticipons quatre domaines de recherche qui pourraient influencer le futur de la gestion de la radioprotection et donc dans lesquels les efforts doivent être poursuivis.

\subsection{L'évaluation du risque}

La très grande majorité des doses reçues par les humains sont dans la gamme des faibles doses, voire dans la gamme des très faibles doses. À ces niveaux de dose, le risque est très faible, à un niveau plus qu'acceptable puisque les études épidémiologiques n'ont pas été en mesure de le démontrer jusqu'à présent. Pour un individu donné, la dose efficace ne peut pas et ne doit pas être utilisée pour l'évaluation du risque (ICRP, 2007). Puisque les principales expositions, c'est à dire le radon et les expositions médicales, sont ciblées sur des organes spécifiques, il semble logique de supposer que le risque porte sur l'organe exposé et non sur le corps entier. Il est par conséquent souhaitable de développer une approche de l'évaluation du risque basée sur les doses à l'organe exposé (dose absorbée et dose équivalente) et sur les modèles de risque ad hoc (UNSCEAR, 2012).

\subsection{La réponse individuelle aux $\mathrm{RI}$ chez l'homme}

Les patients présentant une réponse anormale aux RI devraient être identifiés pour constituer des cohortes aussi larges que possible pour être suivies par des méthodes épidémiologiques, étant donné qu'elles présentent un risque augmenté après exposition aux RI. Dans une démarche inverse, les patients à risque de cancer devraient être étudiés pour rechercher une éventuelle réponse anormale aux RI, comme par exemple les patientes avec un risque familial de cancer du sein ou encore les patients non fumeurs ayant développé un cancer du poumon. Par ailleurs, les patients susceptibles de subir des examens dosants devraient être testés également pour une réponse potentiellement anormale aux RI. Ce dépistage devrait être réalisé en particulier chez les enfants, chez les femmes, avant une radiothérapie ou avant des examens irradiant à répétition, comme les examens de dépistage (par exemple la mammographie). De telles études pourraient permettre de développer des tests prédictifs de la réponse aux RI et pourraient alors devenir une part intégrante de la médecine personnalisée.

\subsection{Les expositions combinées}

De quelle façon faut-il aborder les effets combinés de faibles doses et concentrations de composés génotoxiques, les situations d'expositions combinées étant rencontrées dans la vie de tous les jours pour la majorité de la population? Les effets respectifs de chacun de ces composés pris individuellement au niveau de la concentration environnementale sont probablement impossibles à déterminer. Par conséquent, est-il raisonnable d'essayer de déterminer le risque isolé associé aux très faibles doses RI? Néanmoins, la possible occurrence d'effets supra-additifs de ces expositions combinées reste à déterminer. Cependant, le système de radioprotection apparaît comme un précurseur dans le domaine de la prévention des risques et les principes de justification, d'optimisation et de limitation des doses pourraient être renforcés et étendus à d'autres domaines.

\section{4 Éducation}

Du fait d'un manque de connaissances et de compréhension, la perception par le public et les média des expositions aux RI ne correspond pas aux expositions et risques réels. Caricaturalement, le public et les média imaginent que les expositions naturelles n'existent pas, que les expositions médicales sont bonnes et les expositions dues à l'industrie nucléaire sont mauvaises. La diffusion d'une culture sur les rayonnements ionisants dans les médias, le public et les professionnels de santé est nécessaire pour donner à la radioprotection sa véritable place et non une importance démesurée sur des questions secondaires. Une pédagogie sur la relation linéaire sans seuil est nécessaire. Une approche psychosociale autour de la radioprotection doit être développée. Des personnels ayant des connaissances et des compétences larges dans le domaine de la radioprotection et qui seraient capables de transmettre les bonnes connaissances scientifiques doivent être maintenus. Les estimations des risques liés aux rayonnements ionisants devraient être confrontées à celles liées à d'autres toxiques. De même, les parties prenantes doivent être impliquées de façon plus importante dans les projets éducatifs. La communauté de la radioprotection partage la responsabilité d'une utilisation raisonnée et responsable des finances publiques.

\section{Conclusion}

Cette revue des questions clefs dans le domaine de la radioprotection a permis de mettre en avant des besoins qui balisent le chemin pour le futur de Radioprotection:

- besoin de traiter des expositions les plus importantes et les plus fréquentes et de développer et valider des méthodes permettant de réduire les doses correspondantes, comme par exemple réduire la dose au patient tout en augmentant la qualité des images médicales;

- besoin de développer des recherches en radiobiologie avec les nouvelles méthodes émergentes pour répondre aux 
nombreuses et anciennes questions mais aussi aux nouvelles questions qui n'ont par encore été abordées comme, par exemple, la variabilité de la réponse face à la variété des doses et débits de dose, ou encore la réponse individuelle aux RI;

- besoins d'études épidémiologiques basées sur les cohortes les plus larges possibles mais aussi sur des cohortes ciblées identifiées par l'utilisation de biomarqueurs ;

- besoin d'une dosimétrie optimisée, aussi bien pour les expositions internes que pour les expositions externes;

- besoin de partager les expériences pratiques, en particulier pour l'optimisation de la radioprotection environnementale au cours des opérations de démantèlement afin d'améliorer le cadre légal;

- besoin de nouvelles méthodes d'évaluation des risques pour prendre en compte les expositions combinées aux différents génotoxiques ;

- besoin de maintenir un haut niveau de compétence du personnel en radioprotection et besoin de diffusion d'une culture parmi le public et les média sur les sujets critiques.

La plupart de ces besoins ont également une composante éthique qui n'a pas été abordée dans cette revue mais qui ne doit pas pour autant être oubliée.

Vos contributions dans ces nombreux domaines d'expertise et de recherche pour le futur de la radioprotection sont les bienvenues dans le journal Radioprotection!

\section{Références}

Behjati S, et al. 2016. Mutational signatures of ionizing radiation in second malignancies. Nat. Commun. DOI:10.1038/ncomms12605.

Bosch de Basea M, et al. 2015. EPI-CT: design, challenges and epidemiological methods of an international study on cancer risk after paediatric and young adult CT. J. Radiol. Prot. 35(3): 611-628.

Colin C, et al. 2011. DNA double-strand breaks induced by mammographic screening procedures in human mammary epithelial cells. Int. J. Radiat. Biol. 87: 1103-1112.

European Dose Datamed II Project Report. 2014. European Commission, Radiation Protection no. 180, Part 1/2.

Foray $\mathrm{N}$, et al. 2016. Individual response to ionizing radiation. Mut. Res. 770: 369-386.

Gorin NC, et al. 2006. Consensus conference on European preparedness for haematological and other medical management of mass radiation accidents. Ann. Hematol. 85: 671-679.

Granzotto A, et al. 2016. Influence of nucleoshuttling of the ATM protein in the healthy tissues response to radiation therapy: toward a molecular classification of human radiosensitivity. Int. J. Radiat. Oncol. Biol. Phys. 94(3): 450-460.

ICRP Publication 103. 2007. The 2007 recommendations of the International Commission on Radiological Protection. Ann. ICRP 37(2-4).

IRSN. 2014. Exposition de la population française aux rayonnements ionisants liée aux actes de diagnostic médical en 2012. Rapport IRSN-PRP-HOM no. 2014-6.

IRSN. 2016. La radioprotection des travailleurs. Exposition professionnelle aux rayonnements ionisants en France: bilan 2015. Rapport IRSN-PRP-HOM no. 2016-2.

Joiner MC, et al. 2001. Low-dose hypersensitivity: current status and possible mechanisms. Int. J. Radiat. Oncol. Biol. Phys. 49(2): 379-389.

Joubert A, et al. 2008. DNA double-strand break repair defects in syndromes associated with acute radiation response: at least two different assays to predict intrinsic radiosensitivity? Int. J. Radiat. Biol. 84(2): 107-125.

Herrman TL, et al. 2012. Best practices in digital radiography. American Society of Radiologic Technologists.

Hubert P. 2003. Pour un meilleur usage du risque attribuable en santé environnementale. Environ. Risques Santé 2(5): 266-278.

Lataillade JJ, et al. 2007. New approach to radiation burn treatment by dosimetry-guided surgery combined with autologous mesenchymal stem cell therapy. Regen. Med. 2(5): 785-794.

Laurier D, et al. 2016. The International Nuclear Workers Study (Inworks): a collaborative epidemiological study to improve knowledge about health effects of protracted low-dose exposure. Radiat. Prot. Dosimetry 1-5. DOI: 10.1093/rpd/ncw314.

Leuraud K, et al. 2015. Ionising radiation and risk of death from leukaemia and lymphoma in radiation-monitored workers (INWORKS): an international cohort study. Lancet Haematol. 2: e276-e281.

Little MP, et al. 2012. Systematic review and meta-analysis of circulatory disease from exposure to low-level ionizing radiation and estimates of potential population mortality risks. Environ. Health Perspect. 120: 1503-1511.

Mathews JD, et al. 2013. Cancer risk in 680000 people exposed to computed tomography scans in childhood or adolescence: data linkage study of 11 million Australians. BMJ 346: f2360.

Rothkamm K, Lobrich M. 2003. Evidence of a lack of DNA doublestrand break repair in human cells exposed to very-low X-ray doses. PNAS 100(9): 5057-5062.

UNSCEAR. 2008. Estimates of worldwide average exposures (Vol. 1, Annex B), p. 404.

UNSCEAR. 2012. Attributing health effects to ionizing radiation exposure and inferring risks (Annex A).

Weinberg RA. 2013. The biology of cancer. New York, USA: Garland Science.

Citation de l'article : Bourguignon M, Bérard P, Bertho JM, Farah J, Mercat C. 2017. Radioprotection : quel avenir? Radioprotection 52(1): $13-20$. 